\title{
Anthracologie et paysages forestiers médiévaux : à propos de quatre sites languedociens
}

\author{
In: Annales du Midi : revue archéologique, historique et philologique de la France méridionale, Tome 99, º180, \\ 1987. Paysages, habitat et vie rurale dans le Languedoc médiéval. pp. 397-405.
}

\section{Zusammenfassung}

An vier mittelalterlichen Orten der Ebene des Languedoc weist die Anthracologie Taxone auf, die für die Bergflora charakteristisch sind. Unter den als Erklärung für ihre Anwesenheit auf gestellten Hypothesen ist die wahrscheinlichste die unter gewissen Bedingungen mögliche Fortdauer von mesophilen Hölzern als eiszeitlichen Überresten bis zur Periode des incastellamento in die Schicht der mesomediterranen Vegetation. Diese Interpretation befindet sich in Einklang mit der Gewebestruktur.

\section{Abstract}

In four medieval sites on the Languedoc plain, anthracology has shown the existence of " taxons », species of plants characteristic of mountain flora. Among the hypotheses considered to explain their presence, the most likely is the survival until the incastellamento period, under certain conditions, of mesophilic afforestation, relics of glaciation, in the zone of mesomediterranean vegetation. This interpretation agrees with information found in texts.

\section{Résumé}

Dans quatre sites médiévaux de la plaine languedocienne, l'anthracologie met en évidence des taxons caractéristiques de la flore montagnarde. Parmi les hypothèses envisagées pour expliquer leur présence, la plus vraisemblable est le maintien, sous certaines conditions, de boisements mésophiles reliques glaciaires jusqu'à la période d'incastellamento dans l'étage de végétation mésoméditerranéen. Cette interprétation est en accord avec les données textuelles.

Citer ce document / Cite this document :

Durand Aline, Vernet Jean-Louis. Anthracologie et paysages forestiers médiévaux : à propos de quatre sites languedociens. In: Annales du Midi : revue archéologique, historique et philologique de la France méridionale, Tome 99, º180, 1987. Paysages, habitat et vie rurale dans le Languedoc médiéval. pp. 397-405.

doi : 10.3406/anami.1987.2160

http://www.persee.fr/web/revues/home/prescript/article/anami_0003-4398_1987_num_99_180_2160 


\section{Aline DURAND et Jean-Louis VERNET*}

\section{ANTHRACOLOGIE ET PAYSAGES FORESTIERS MÉDIÉVAUX :}

\section{A PROPOS DE QUATRE SITES LANGUEDOCIENS}

L'histoire de la couverture végétale méditerranéenne demeure mal connue pour l'époque médiévale. En dehors de quelques études pionnières restées isolées ${ }^{1}$, on ne sait presque rien du type et de la composition de l'environnement végétal dans lequel vivaient les hommes des IXe-XIVe siècles. Pourtant, la plupart des historiens médiévistes considèrent depuis longtemps que c'est entre la fin du $\mathrm{X}^{\mathrm{e}}$ et le milieu du XIIe siècle qu'ont eu lieu les modifications les plus radicales du paysage forestier du Sud de la France. En revanche, les écologues et géomorphologues sont dans leur ensemble d'accord pour penser que, dès la protohistoire, les paysages sont semblables aux actuels. Les principales étapes de mise en place de la végétation méditerranéenne, bien connues aujourd'hui grâce à l'anthracologie et à la palynologie, montrent que les processus de défrichement débutent dès le Néolithique et s'accélèrent surtout au Chalcolithique, Bronze et Fer. Dans le Midi, qui appartient pour l'essentiel au climat méditerranéen subhumide d'Emberger ou à l'étage mésoméditerranéen d'Ozenda $^{2}$, les boisements mésophiles ${ }^{3}$ constitués principalement de chênes pubescents disparaissent entre 4000 et $2000 \mathrm{BP}$. La garrigue, formation régressive de ligneux bas date ainsi de ces périodes. Or, le développement récent de l'archéologie médiévale dans le Languedoc oriental rend directement possible une étude de la végétation à partir des restes de bois carbonisés pour cette époque.

Dans cette perspective, la flore des gisements médiévaux de Montagnac (880-1140 A.D., $1040 \pm 60$ apr. J.C., sarcophage monolithique), Lunel-Viel

\footnotetext{
* A. Durand, 26, rue Pierre-et-Marie-Curie, 75005 Paris ; J.-L. Vernet, Institut des Sciences de l'Evolution, U.A. 327, place E. Bataillon, 34060 Montpellier Cedex.

1. J.-P. BARRY et E. Le Roy LADURIE, Histoire agricole et phytogéographie, dans Annales E.S.C., t. XVII, 1962, p. 434-447 ; G. Demians d'ARchimbaud, Les fouilles de Rougiers, Valbonne, 1980. p. 405-407; Ch. Higounet, Les forêts de l'Europe occidentale du Ve au XI' siècle, dans Settimani di studio del Centro italiano di studi sull' alto medioevo, XIII, Agricoltura e mondo rurale in Occidente nell' alto medioevo, Spoleto, 1965 (1966), p. 343-398; T. SCLAFERT, Cultures en HauteProvence. Déboisements et pâturages au Moyen Age, Paris, 1959 ; P. Tucoo-CHALA, Forêts et landes en Béarn au XIVe siècle, dans Annales du Midi, 1955, p. 248-251.

2. P. OzENDA, Sur les étages de végétation dans les montagnes du bassin méditerranéen, dans Documents de cartographie écologique, vol. XVI, Grenoble, 1975, p. 1-32.

3. Plantes mésophiles : végétaux croissant dans des conditions moyennes d'humidité par opposition aux xérophiles bien adaptées à la sécheresse et aux hygrophiles dont l'écologie exige la présence permanente d'eau.
} 


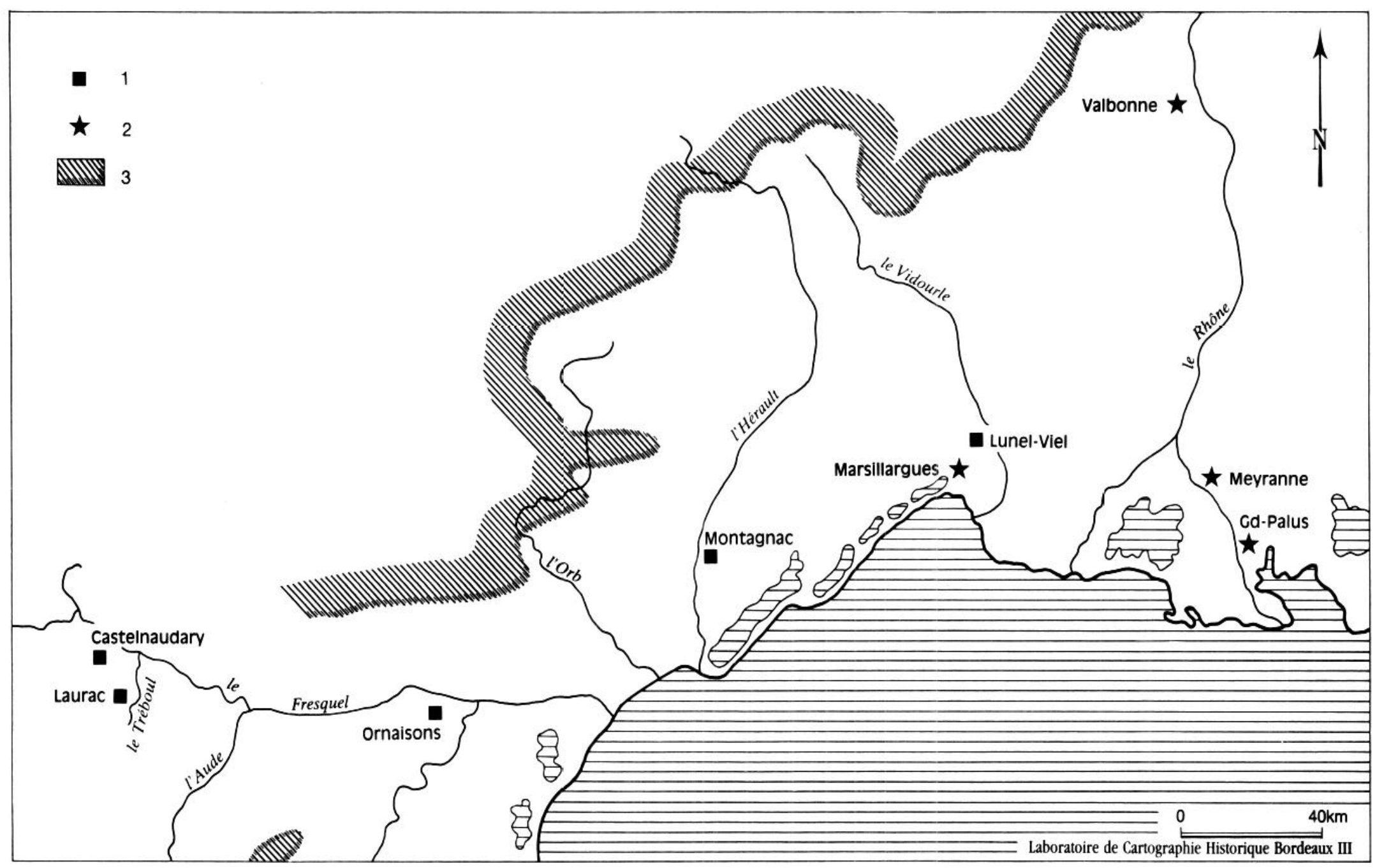

Carte des gisements.

1 : Sites anthracologiques. 2 : Sites à taxons mésophiles. 3 : Limite inférieure du hêtre $(800 \mathrm{~m})$. 
(XI ${ }^{e}-X{ }^{e}$ siècles, silos à grains), Laurac ( $\mathrm{X}^{\mathrm{e}}-\mathrm{XII}{ }^{\mathrm{e}}$ siècles, silo à grains) et Castelnaudary (XIV $\mathrm{XV}^{\mathrm{e}} \mathrm{XV}^{\mathrm{e}}$ siècles, fossé) ${ }^{4}$ est une contribution à cette histoire.

A Montagnac, la liste des espèces suivantes a été établie : hêtre, sapin, noisetier, frêne élevé, orme champêtre, peuplier noir ainsi que chêne pubescent, frêne oxycarpe, laurier sauce et chêne vert. La moitié de ces taxons n'est pas caractéristique de l'actuelle flore méditerranéenne que seuls le frêne oxycarpe, le chêne pubescent, le laurier et le chêne vert représentent. Hêtre, sapin, noisetier ou frêne élevé sont localisés de nos jours sur les bordures cévenoles ou caussenardes, vers $700-800 \mathrm{~m}$ pour le hêtre.

A I unel-Viel, la flore est assez voisine de la précédente, avec cependant une empreinte méditerranéenne plus marquée : chêne vert ou chêne kermès (LVI-2), pin d'Alep (LV2), pin pignon (LV2), genévrier de type de Phénicie (LV2), vigne (LV1-2), cyprès (LV2), pommier (LV1) et bruyère (LV2). Les taxons mésophiles ou montagnards sont le sapin (LV1-2), le frêne élevé (LV2) et l'orme (LV1).

A Laurac, hêtre, noisetier et chêne pubescent ont seuls été rencontrés dans un contexte actuellement méditerranéen.

Enfin, le site plus tardif de Castelnaudary permet aussi de retrouver la dualité espèces mésophiles-espèces méditerranéennes avec un ensemble constitué de hêtre, sapin, noisetier, chêne à feuillage caduc accompagné de chêne vert ou de chêne kermès, châtaignier, mûrier noir, vigne, cyprès, cerisier, prunier ou prunellier et genévrier (type de Phénicie).

Ainsi, dans ces quatre gisements, nous avons pu mettre en évidence des taxons mésophiles (sapin et hêtre). Leur présence principalement au Moyen Age central mérite que l'on s'y attarde un peu. Elle pose le problème de la coexistence au sein de forêts ou de lambeaux de forêts méditerranéennes d'espèces appartenant à des paysages bien différents d'altitudes supérieures. Plusieurs explications peuvent être avancées :

- les cours d'eau auprès desquels ont été trouvés nos charbons ont charrié lors des crues, depuis les contreforts montagneux jusque dans les plaines, des branches et bois morts qui ont fait l'objet d'un ramassage par droit d'usage ;

- la société rurale des $\mathrm{X}^{\mathrm{e}}-\mathrm{XIII}{ }^{\mathrm{e}}$ siècles a mis en place des circuits économiques qui impliquent un transfert de bois des marges minervoises et caussenardes vers les basses vallées de façon suffisamment régulière pour être perçu au sein des restes archéologiques;

- enfin des boisements mésophiles survivent de manière exceptionnelle au sein même du complexe écologique méditerranéen de basse altitude.

4. Nous remercions M. Dauzat (Castelnaudary et Laurac), C. Pellecuer (Montagnac), C. Raynaud (Lunel-Viel) qui unt bien voulu nuus conffier l'étude de leur mobilier anthracologique, et G. Dclibrias, du Centre des faibles radioactivités de Gif-sur-Yvette, qui a effectué la datation au $\mathrm{C}^{14} \mathrm{des}^{-}$ échantillons de Montagnac (Réf. Gif-6637). 


\begin{tabular}{|c|c|c|c|c|c|c|c|c|c|c|}
\hline & & & nthr & colo & & & & $\stackrel{\widehat{n}}{\stackrel{0}{=}}$ & $\widehat{n}$ & \\
\hline & 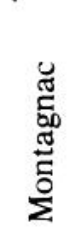 & 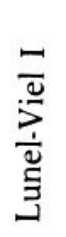 & 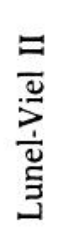 & 苋 & 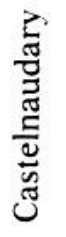 & $\begin{array}{l}\tilde{D} \\
\frac{\mathscr{n}}{\widetilde{E}} \\
0\end{array}$ & 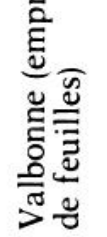 & 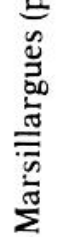 & 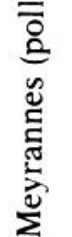 & 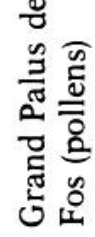 \\
\hline Abies alba & + & + & + & & + & + & & & & + \\
\hline Acer sp. & & & & & & + & & + & & \\
\hline Alnus glutinosa & & & & & & & & + & + & + \\
\hline Angiosperme indéterminée & & & + & & & & & & & \\
\hline Arbutus unedo & & & & & & + & & & & \\
\hline Arundo donax & & & & & & + & & & & \\
\hline Betula sp. & & & & & & & & $\bullet$ & $\bullet$ & + \\
\hline Buxus sempervirens & & & & & & + & & + & & \\
\hline Castanea sativa & & & & & + & & & + & & \\
\hline Celtis australis & & & & & & & & & $\bullet$ & \\
\hline Corylus avellana & + & & & + & + & & + & $\bullet$ & $\bullet$ & + \\
\hline Cupressacée indéterminée & & & + & & & & & + & & + \\
\hline Cupressus sempervirens & & & + & & + & & & & & \\
\hline Ericacée indéterminée & & & + & & & & & & + & + \\
\hline Erica arborea-multiflora & & & + & & & + & & & & \\
\hline Erica arborea & & & & & & & & + & & \\
\hline cf. Evonymus europeus & & & & & & + & & & & \\
\hline Fagus silvatica & + & & & + & + & + & + & $\bullet$ & $\bullet$ & + \\
\hline Fraxinus $s p$ & & & & & & & & & & + \\
\hline Fraxinus excelsior & + & & + & & & & & & & \\
\hline Fraxinus oxycarpa & + & & & & & + & & & & \\
\hline Genista scorpius & & & & & & + & & & & \\
\hline Gymnosperme indéterminée & & & + & & & & & & & \\
\hline Juniperus type phoenicea & & & + & & + & & & & + & \\
\hline Juglans $s p$. & & & & & & & & + & $\bullet$ & $\bullet$ \\
\hline Lauris nobilis & + & & & & & & & & & \\
\hline Morus nigra & & & & & + & & & & & \\
\hline Olea europea & & & & & & + & & + & $\bullet$ & + \\
\hline Phillyrea sp. & & & & & & & & + & + & \\
\hline Phillyrea cf. media & & & & & & + & & & & \\
\hline Pinus sp. & & & + & & & & & & + & + \\
\hline Pinus cf. halepensis & & & + & & & & & & & \\
\hline Pinus halepensis & & & + & & & + & & & & \\
\hline Pinus pinea & & & + & & & & & & & \\
\hline Pirus malus & & + & & & & & & & & \\
\hline Pistacia $s p$. & & & & & & & & & & $\bullet$ \\
\hline Populus nigra & + & & & & & & & & & \\
\hline Populus tremula & & & & & & & + & & & \\
\hline Prunus cf. cerasus & & & & & + & & & & & \\
\hline Prunus cf. insititia & & & & & & + & & & & \\
\hline Prunus spinosa-domestica & & & + & & + & & & & & \\
\hline Quercus sp. & & & & & & & & + & & \\
\hline Quercus ilex & + & & + & & & + & & & & \\
\hline Quercus type ilex & & + & & & & & & & & \\
\hline Quercus feuil. caduc & + & & & + & + & + & + & & + & + \\
\hline Salicacée indéterminée & & & & & + & & & & & \\
\hline Sorbus latifolia & & & & & & & + & & & \\
\hline Ulmus campestris & + & + & & & & + & + & & & \\
\hline Vitis vinifera & & + & + & & + & + & & + & & \\
\hline
\end{tabular}

+ présence notoire (fort \%)

- présence significative (pour les polleris)

Comparaison des flores médiévales à taxons mésophiles. 


\section{GLOSSAIRE}

Abies alba

Acer sp.

Alnus glutinosa

Arbutus unedo

Arundo donax

Betula sp.

Buxus sempervirens

Castanea sativa

Celtis australis

orylus avellana

-upressacée indéterminée

Cupressus sempervirens

Ericacée

Erica arborea-multiflora

Erica arborea

cf. Evonymus europeus

Fagus silvatica

Fraxinus sp.

Fraxinus excelsior

Fraxinus oxycarpa

Genista scorpius

Hedera helix

Ilex aquifolium

Juniperus type phoenicea

Juglans $s p$.

Lauris nobilis

Morus nigra

Olea europea

Phillyrea sp.

Phillyrea cf. media

Pinus sp.

Pinus cf. halepensis

Pinus pinea

Pirus malus

Pistacia sp.

Populus nigra

Populus tremula

Prunus cf. cerasus

Prunus cf. insititia

Drunus spinosa-domestica

Quercus sp.

Quercus ilex

Quercus type ilex

Quercus à feuillage caduc

Quercus sessiliflora

Salicacée

Sorbus aria

Sorbus latifolia

Sorbus torminalis

Ulmus campestris

Vitis vinifera
Sapin blanc

Erable

Aulne glutineux

Arbousier

Canne de Provence

Bouleau

Buis

Châtaignier

Micocoulier

Noisetier commun

Famille des Cupressacées

Cyprès sempervirent

Famille des Ericacées

Bruyère arborée ou multiflore

Bruyère arborée

cf. Fusain d'Europe

Hêtre

Frêne

Frêne élevé

Frêne à fruits rétrécis

Genêt scorpion

Lierre

Houx

Genévrier type de Phénicie

Noyer

Laurier sauce

Mûrier noir

Olivier

Alavert

Alavert à feuille moyenne

Pin

Pin d'Alep

Pin pignon

Pommier

Pistachier

Peuplier noir

Peuplier tremble

Prunier of. Cerisier

Prunier cf. Mirabellier

Epine noire ou prunier domestique

Chêne

Chêne vert

Chêne de type Chêne vert

Chêne à feuillage caduc (type chêne pubescent)

Chêne sessiliflore

Famille des Salicacées

Alouchier (Alisier blanc)

Alisier à larges feuilles

Alisier (Sorbier torminal)

Orme champêtre

Vigne 
Cette dernière hypothèse est celle qui paraît a priori la moins probable car elle semble remettre en cause les données classiques d'étagement de la végétation méditerranéenne. Cependant, le hêtre peut encore à l'heure actuelle se rencontrer plus bas que sa limite normale, par exemple à $500 \mathrm{~m}$ d'altitude sur le versant sud du Causse du Larzac, au contact même du chêne vert. La hêtraie occupe alors les faciès les plus profonds, les plus mésophiles, c'est-à-dire les plus " montagnards ". Il est donc nécessaire d'approfondir la question en comparant nos résultats à ceux de la documentation paléoécologique médiévale existante pour d'autres sites méditerranéens de plaine : Ornaisons 5 , Valbonne6, Marsillargues ${ }^{7}$, Meyranne et les Grands Paluds de Fos ${ }^{8}$.

L'intérêt de ces cinq sites doit être souligné : ils in tègrent respectivement des résultats obtenus sur des charbons de bois (Ornaisons), des feuilles fossiles (Valbonne) et des pollens (Marsillargues, Meyranne et les Grands Paluds). Dans ces conditions, la diversité des restes et des types de dépôts (couches archéologiques, tourbières de lagunes, alluvions locales) rend caduque la première explication. Envisageable pour les pollens et partiellement pour les charbons, elle est tout à fait impossible pour les tufs de Valbonne. De plus, les charbons de nos gisements proviennent de structures archéologiques variées (fossés, silos à grains, sarcophages, etc.), ce qui va dans le même sens.

A Ornaisons, la flore du très haut Moyen Age s'avère bien différente des taxons mis à jour pour les périodes antérieures. Le chêne pubescent se raréfie alors que le chêne vert est bien représenté ; l'olivier et la vigne montrent l'extension des cultures ; l'aggravation de la dégradation se traduit par l'augmentation de formations basses de type garrigue; elle profite au pin d'Alep qui se comporte comme un véritable bouche-trou. C'est dans un tel contexte que hêtres et sapins sont représentés, les premiers surtout.

La forêt de Valbonne, près de Pont-Saint-Esprit entre 75 et $300 \mathrm{~m}$ d'altitude, a fourni des travertins à empreintes de feuilles datés par le $\mathrm{C}^{14}$ de $870 \pm 50 \mathrm{BP}$ soit entre 1035 et $1255 \mathrm{apr}$. J.C. Ils sont donc antérieurs, voire à peine contemporains de la fondation du monastère en 1204. Les feuilles recueillies et étudiées par $\mathbf{M}$. Farizier appartiennent aux espèces suivantes : hêtre $(90 \%)$, accompagné de sorbier à feuille large (hybride torminalis et aria), orme champêtre, chêne à feuillage caduc, peuplier tremble, noisetier. Il est remarquable dans ce cas que les taxons mésophiles de la flore médiévale soient également présents dans la flore actuelle. En effet, le milieu

\footnotetext{
5. J.-L. VERNET, inédit.

6. M. FARIZIER, Recherches sur les macroflores des tufs quaternaires du Sud de la France, Dipl. EPHE, USTL, Montpellier, 1980.

7. N. PlanchaIs, Palynologie lagunaire de l'étang de Mauguio. Paléoenvironnement végétal et évolution anthropique, dans Pollen et Spores, Vol. XXIV, $\mathbf{n}^{\circ} 1$, p. 93-118.

8. H. TRIAT-LaVal, Contribution pollenanalytique à l'histoire tardi-et post-glaciaire de la végétation de la basse vallée du Rhône, thèse Etat, Aix-Marseille-III, 1978 (1979).
} 
végétal actuel est totalement atypique. S'il appartient globalement à l'étage climatique mésoméditerranéen supérieur et à la série de végétation " forêt mixte à chêne vert et chêne pubescent ", ce qui est normal pour ces collines au sud du confluent Rhône-Ardèche, on y rencontre aussi des peuplements importants de hêtres qui y prospèrent en bénéficiant d'un substrat favorable (marnes et sables Crétacé) et d'une topographie contribuant à la conservation de l'eau souvent à fleur de sol (collines de 240 à $300 \mathrm{~m}$ entrecoupées de combes). Le cortège floristique de cette forêt est cependant appauvri par rapport aux stations montagnardes habituelles, ce qui est à rapprocher à la fois des observations effectuées à partir des feuilles fossiles et de nos résultats anthracologiques : le hêtre n'est accompagné que de sorbier torminal, d'orme champêtre, de lierre, chêne sessiliflore, alisier blanc et houx. Une forêt mésophile peut donc très bien exister et se maintenir dans l'étage mésoméditerranéen supérieur si des conditions édaphiques satisfaisantes compensent la longue période de sécheresse estivale régnant dans cet étage. Or ces conditions sont réunies le long des thalwegs des cours d'eau méditerranéens pérennes bordant nos gisements : Hérault près de Montagnac, Vidourle pour Lunel-Viel, Fresquel à Castelnaudary et Tréboul pour Laurac. Elles sont réunies aussi à proximité des lagunes littorales auprès desquelles ont été dressés les diagrammes polliniques.

Ainsi, à Marsillargues, alors que toutes les périodes antérieures (depuis le début du diagramme, $4720 \mathrm{BP}$ ) étaient marquées par une très forte représentation des arbres forestiers mésophiles, surtout le hêtre, à égalité avec les espèces méditerranéennes, on constate pour le Moyen Age central leur quasi-régression ; au même moment, les espèces cultivées telles l'olivier, le noyer et le châtaignier sont très fortement représentées. De même, on observe, malgré l'absence de datations au carbone 14 pour les périodes les plus récentes, de nettes concordances entre les diagrammes établis pour la basse vallée du Rhône et celui de Marsillargues. L'auteur, H. Triat-Laval, y rencontre des pollens de hêtre et de sapin dans les tourbes ou des alluvions fluviatiles depuis l'holocène. On peut constater qu'ils y persistent jusque dans les périodes historiques. Elle envisage pour le Tardiglaciaire que les vallées aient pu jouer un rôle refuge dans le maintien du hêtre. Le raisonnement peut $a$ fortiori s'appliquer à la période médiévale.

Dans l'état actuel de la recherche historique et de nos connaissances des sources régionales languedociennes antérieures au XIII siècle, aucun indice n'est venu confirmer la seconde des hypothèses proposées 9 . Au contraire, toutes les données écologiques concourent à envisager l'existence pour l'époque médiévale de hêtraies ou de chênaies-hêtraies dans les sites les plus

9. Il existe bien des mentions de bois transportés des Cévennes et des Causses dans la basse plaine, mais elles sont généralement postérieures à 1250 et mentionnent toujours des essences spécifiques, comme le châtaignier, qui sont réservées à des constructions importantes telles les navires du roi saint Louis. En aucun cas elles ne concernent la société paysanne. 
mésophiles, lagunaires ou terrasses alluviales, au sein de l'étage méditerranéen. Le sapin a-t-il pu faire partie du paysage ? La question reste posée. Il faut rappeler que c'est surtout au cours du Néolithique et de l'âge des Métaux que l'essentiel des hêtraies de nos régions se mettent en place. Le hêtre se développe ainsi dans le Sud de la France à un moment (post 3600 BP) où l'homme étend son emprise sur l'environnement. En d'autres termes, les hêtraies et les hêtraies-sapinières apparaissent à une époque, le Néolithique, où les processus d'anthropisation se révèlent, et s'amenuisent ou disparaissent au Moyen Age avec la forte anthropisation du paysage. En effet, le hêtre n'apparaît dans les charbons de bois des sites mésoméditerranéens récents qu'au Moyen Age, justement au moment où sa représentation pollinique diminue.

Tout ceci ne peut avoir qu'une signification : les forêts mésophiles riveraines n'ont été défrichées qu'à l'époque médiévale et plus précisément entre le $X^{e}$ et la fin $d u X I I^{e}$ siècle. Les données historiques les plus récentes concernant le Languedoc oriental viennent étayer cette interprétation. Pour la région biterroise, M. Gramain-Derruau10, F. Cheyette et C. DuhamelAmado 11 ont bien montré la constitution progressive à partir de 900-960 de nouveaux terroirs le long des rivières. La mise en valeur de ces terres alluvionnaires sans doute plus difficiles à travailler que la terra rossa habituelle est désormais possible grâce au développement des techniques agraires : la diffusion du moulin à paissière, qui régularise les débits des rieux et cours d'eau, permet l'irrigation systématique des cultures et prairies naturelles. De plus l'outillage se perfectionne : l'emploi d'un fer de meilleure qualité et l'utilisation massive du ferrage des bêtes permettent au Moyen Age le travail de sols plus lourds, tels ceux des bords des rivières négligés jusqu'ici faute d'une maîtrise technique suffisante.

Les hypothèses déjà avancées par $\mathbf{N}$. Planchais et $\mathrm{H}$. Triat-Laval pour le Tardiglaciaire et E. Bazile-Robert12 pour le Paléotithique supérieur se trouvent donc confortées et démontrées également pour les périodes historiques. Ainsi, dans le Languedoc oriental, non seulement la silva se fixe-t-elle définitivement entre les $\mathrm{X}^{e}$ et XIII ${ }^{e}$ siècles avec la fin de tout défrichement de zrande ampleur, mais encore prend-elle l'aspect qu'elle a gardé jusqu'à nos jours. C'est au Moyen Age que les reliques des glaciations disparaissent de la végétation méditerranéenne de basse altitude, entraînant ainsi un certain appauvrissement des paysages forestiers. Chronologiquement, ce changement d'ordre floristique semble bien correspondre aux transformations de l'habitat et des structures agraires ayant affecté le Languedoc oriental à

10. M. Gramain-DeRRUAU, Villages et communautés villageoises en Bas-Languedoc occidental (vers 950-vers 1350) : l'exemple biterrois, thèse Etat, Paris-I, 1979, notamment p. 247-262.

11. F. Cheyette et C. Duhamel-Ayado, Organisation d'un terroir et d'un habitat concentré : un xemple languedocien, dans Habitats fortifiés et organisation de l'espace en Méditerranée médiéale, Table ronde de Lyon (1982), Lyon, 1983 p. 35-44.

12. E. BAzILE-RoBert, Flore et végétation du Sud de la France pendant la dernière glaciation d'après l'analyse anthracologique, thèse $3^{e}$ cvele. USTL, Montpellier, 1979. 
partir de l'an Mil13. Il serait alors l'un des signes les plus visibles de la mise en place de nouveaux systèmes de cultures, c'est-à-dire de nouveaux rapports ager-saltus-silva, et d'une nouvelle organisation de l'espace rural. L'incastellamento n'aurait pas seulement bouleversé le tissu humain, il aurait également transformé les rapports de l'homme et de son cadre végétal.

13. M. Gramain-Derruau, op. cit., et M. Dauzat, Les mottes castrales du Lauragais : notes préliminaires, dans Le Lauragais. Histoire et archéologie, LIVe Congr. Féd. hist. Languedoc méditerranéen et Roussillon, Montpellier, 1983, p. 73-87 ; M. PASSELAC, Nouvelles découvertes et observa. tions aériennes des sites médiévaux en Languedoc, dans Archéologie du Midi médiéval, t. II, 1984, p. 5-14. 